\title{
Eclipsing Binaries Within Visual Ones: Prospects of Combined Solution
}

\author{
Petr Zasche \\ Astronomical Institute, Faculty of Mathematics and Physics, Charles University Prague, \\ CZ-180 00 Praha 8, V Holešovičkách 2, Czech Republic \\ email: zasche@sirrah.troja.mff.cuni.cz
}

\begin{abstract}
The study of eclipsing binaries as members of multiple systems can provide us important information about their origin, evolution, mutual inclination of the orbits, independent distance and mass determination, as well as the stellar multiplicity in general. We are carrying out a long-term photometric monitoring of several eclipsing binaries within the visual multiples and, besides the complete light curves, we are trying to detect the period changes due to the orbital motion around a common barycenter.

Systems like DN UMa, V819 Her, LO Hya, or VW Cep are typical examples of eclipsing binaries orbiting around the barycenter of the multiple system, while their respective periods are on the order of years or decades. However, the expected period variation is only hardly detectable and there is still uncertainty about which of the components is the eclipsing one. Precise spectroscopy would be of great benefit, but detecting the changes in the gamma velocity is still problematic, and spectral disentangling of such complicated systems like sextuple VV Crv (periods 1.46, 3.14, and 44.51 days) is also rather difficult. However, the detection of the changing depths of the eclipses in the latter system would be interesting.
\end{abstract}

Keywords. (stars:) binaries: eclipsing, (stars:) binaries: spectroscopic, (stars:) binaries: visual, stars: fundamental parameters

There are still known only a few systems where an eclipsing binary is a member of a more complex visual multiple system, and exhibits periodic modulation of its orbital period as a result of orbital motion around a common barycenter. There are only eight such systems known nowadays - i Boo, VW Cep, KR Com, V772 Her, V819 Her, QS Aql, $\zeta$ Phe, and V505 Sgr. Such systems are usually rather close to the Sun (often $<100 \mathrm{pc}$ ), and their angular separations are on the order of a few mas only. There are many possible effects which can be studied and moreover there are also several physical constraints which have to be satisfied in such systems (e.g. stability criteria as a function of ratio of long and short periods).

Three different systems studied recently (namely DN UMa, VV Crv, and V2083 Cyg) indicate that there could be hidden components in these multiples, but each of the additional components was discovered by a different method. Detailed analyses will be published elsewhere in a separate paper. Discovering additional components in a particular system shifts the object to even higher multiplicity and also affects the statistics of such systems. Eggleton \& Tokovinin (2008) published a study of multiplicity among bright (well-studied) systems. They presented a correlation between the number of systems of a particular multiplicity versus the number of components in the system. As we have pointed out, such statistics are nowadays still very incomplete and many systems are of higher multiplicity than listed in catalogues.

Radial velocity residuals, spectral disentangling, or long-term photometric monitoring of these systems is very fruitful, but to obtain a reliable and complete picture of the system is still difficult. A study of dynamical effects in these systems should be of interest, for instance a slow precession of the orbits or changing the inclination between them. 
In some of the systems, discovering additional components could be a less difficult task due to several reasons. One of them is quite surprising, but it is the fact that most of these stars are relatively bright. Very bright stars $(<6$ mag) are photometrically observed only very rarely nowadays due to larger telescopes and CCD detectors used (these targets easily saturate the detectors). New observations can reveal additional components simply by observing the minima of eclipsing binaries and detecting the period changes. Many of the bright stars are very neglected nowadays. Furthermore, discovering additional components has other aspects, for example most of the detected components are of similar spectral types as the eclipsing pair itself. This is understandable, because it is much more difficult to discover a low-mass companion near a pair of B stars.

Another issue is the detection method of such bodies. As an illustrative example we present here three different cases, where three different methods of detection were applied:

- DN UMa - This well-known eclipsing binary is a member of a more complex multiple system with two distant components $\left(63^{\prime \prime}\right.$ and $\left.4^{\prime \prime}\right)$. The light curve (LC) was analyzed by García \& Giménez (1986), and the radial velocity curve (RV) by Popper (1986). Moreover, another component is orbiting around the eclipsing pair with period about 118 yr. Many observations of minima times for the EB pair obtained during the last 30 years were used for a period analysis. After subtracting the $118 \mathrm{yr}$ trend, we discovered another variation with period about 640 days only, which can be attributed to another component in the system. Therefore, we have a sextuple star system.

- VV Crv - This rather neglected multiple system was studied by Massarotti et al. (2008) by means of radial velocities. Two different spectroscopic binaries were discovered (periods 44.5 and 1.46 day). Furthermore, two other distant visual components are present in the system $\left(60^{\prime \prime}\right.$ and $\left.5^{\prime \prime}\right)$. And finally, there is also a 3.14 day eclipsing pair, which cannot be related to either of the spectroscopic periods. Therefore, the system is a typical hierarchical sextuple. Moreover, a very favorable configuration of the system allows us to hope to detect a change of inclination (i.e. short nodal period).

- V2083 Cyg - A typical triple system consisting of an EB pair on a wide orbit with period of about $372 \mathrm{yr}$ (Seymour et al. 2002). However, our new detailed analysis of LC, RV and visual orbit reveals that the system is probably of higher multiplicity. We found that the total mass of the system as derived from the visual orbit is much higher than the one found from the LC+RV analysis. A possible solution is to move the system closer to the Sun, but this leads to a value of parallax well outside of the error bars of the Hipparcos data. Another possible solution is that the distant component is also a double.

We can also study the mutual inclinations of the orbits in these binaries (eclipsing-pair orbit versus visual-pair orbit). As pointed out by Zakirov (2008), the inclination angles are not randomly oriented and such systems tend to be coplanar. This indicates something about a common origin of the system. However, we found a completely different picture. The mutual inclinations are high $\left(>30^{\circ}\right)$ and for V2083 Cyg the orbits are almost perpendicular to each other $\left(i_{2-3}=82^{\circ}\right)$.

Acknowledgements: Supported by the Czech Science Foundation grant P209/10/0715.

\section{References}

Eggleton, P. P. \& Tokovinin, A. A. 2008, MNRAS, 389, 869

García, J. M. \& Giménez, A. 1986, Ap\&SSS, 125, 181

Massarotti, A., Latham, D. W., Stefanik, R. P., \& Fogel, J. 2008, AJ, 135, 209

Popper, D. M. 1986, PASP, 98, 1312

Seymour, D. M., Mason, B. D., Hartkopf, W. I., \& Wycoff, G. L. 2002, AJ, 123, 1023

Zakirov, M. M. 2008, Kinematics and Physics of Celestial Bodies, 24, 25 